\title{
LA PESQUERÍA COMERCIAL DE LORETO CON ÉNFASIS EN EL ANÁLISIS DE LA RELACIÓN ENTRE CAPTURA Y ESFUERZO PESQUERO DE LA FLOTA COMERCIAL DE IQUITOS, CUENCA DEL AMAZONAS (PERÚ)
}

\author{
Salvador Tello*, Peter Bayley ${ }^{* *}$
}

\section{RESUMEN}

La información utilizada en este estudio fue recopilada por el Instituto de Investigaciones de la Amazonía Peruana (IIAP), la Dirección Regional de Pesquería de Loreto y la Asociación de Pescadores de Loreto. Para ser analizada, dicha información se separó en desembarques totales de la región Loreto y desembarques de la flota pesquera comercial de Iquitos. Los resultados indican que las especies grandes están siendo reemplazadas en los desembarques por especies más pequeñas, de alto rendimiento y de menor precio. Esto se debe a la mayor intensidad de pesca y a los cambios en las artes de pesca. Asimismo, se encontró evidencia de sobrepesca de crecimiento en algunas especies de grandes bagres y boquichico. No obstante ello, los rendimientos comerciales encontrados en similares niveles de esfuerzo han permanecido casi constantes a pesar de los cambios en la composición de las capturas detectadas en los últimos años. Para el área de estudio, se determinó un esfuerzo de 0,3 pescadores por $\mathrm{km}^{2} \mathrm{y}$ un rendimiento de $1,4 \mathrm{~kg} \mathrm{ha}^{-1}$.

Palabras clave: Pesquería comercial, bagre, boquichico, capturas.

\begin{abstract}
The information used in this study was collected by the Peruvian Amazon Research Institute, Regional Fishery Government of Loreto and the Loreto's Fishers Association, and was analyzed separating it in total disembarks of Loreto and disembarks of the Iquito's Commercial Fisher Fleet. The results indicate that large species are being replaced in the disembarks for smaller species, of high performance and of smaller

* Instituto de Investigaciones de la Amazonía Peruana (IIAP). Programa de Ecosistemas Acuáticos. Correo electrónico: dirpea@rail.org.pe

** Oregon State University (OSU). Correo electrónico: bayleyp@ucs.orst.edu
\end{abstract}


price as consequence of a greater intensity of fishing and changes in the arts of fishing. Likewise, was found evidence about growth overfishing in some species of large «bagres» and «boquichico». Nevertheless it, the commercial performances to similar levels of effort found have remained almost constant in spite of the changes in the composition of the captures detected in the last years. For the area of study, was determined an effort of 0,3 fishers by $\mathrm{km}^{2}$, with a performance of $1,4 \mathrm{~kg} \mathrm{has}^{-1}$.

Key words: Comercial fishing, bagre, boquichico, captures.

\section{INTRODUCCIÓN}

En la Amazonía Peruana, la pesca es una importante fuente de abastecimiento de proteína animal y de ingresos económicos para el poblador ribereño, debido a que el pescado es el principal componente de la dieta familiar y a que las capturas totales están valorizadas en 80 millones de dólares anuales. Dentro de la pesca de consumo, la pesca de subsistencia y la comercial son las de mayor relevancia porque cerca del $75 \%$ y del $25 \%$ del volumen de pescado desembarcado cada año en la región son capturados por ambas pesquerías, respectivamente.

El manejo y desarrollo de la pesquería en la Amazonía no es una tarea fácil. Las condiciones ecológicas en las zonas de várzea y la relación entre las planicies inundables y los ríos son muy complejas. Este hecho dificulta cualquier acción de manejo. A esto se suman el carácter multiespecífico de las pesquerías, la ausencia de conocimientos sobre la bioecología de especies de consumo y la dificultad de identificar stocks (Gulland,1982; Turner, 1985; Bayley y Petrere, 1989).

Una forma de estimar el rendimiento de una pesquería multiespecífica es a través de la aplicación el modelo de Graham-Schaefer o de la captura por unidad de esfuerzo (CPUE), que relaciona la captura total, como peso total, de todas las especies capturadas con el esfuerzo total desplegado. Este modelo ha sido aplicado en ambientes tropicales (Welcomme, 1975), en la cuenca amazónica (Petrere, 1983; Bayley y Petrere, 1989; Montreuil y Tello 1990, 1997) y es también utilizado con los desembarques de la flota comercial de Iquitos en el presente estudio. El propósito de este reporte es proporcionar información sobre la flota pesquera comercial de Loreto para el manejo, desarrollo y conservación de la pesquería en el Amazonas peruano. 


\section{MATERIALES Y MÉTODOS}

\section{Área de Estudio}

El área de estudio está localizada en la Amazonía Peruana y cubre los ríos Ucayali y Amazonas, que son las cuencas de mayor importancia pesquera. Esta región tiene características de zona tropical húmeda. Sus temperaturas van desde los 20 hasta los $33{ }^{\circ} \mathrm{C}$ y sus precipitaciones anuales varían desde 2000 hasta $3000 \mathrm{~mm}$.

Las planicies inundables más productivas de la Amazonía Peruana están localizadas en el área de estudio, en particular, aquellas asociadas con el Ucayali.

Las condiciones hidrológicas producen «pulsos» de inundación que son los responsables de la alta producción pesquera. Se encuentra en esta cuenca excelentes niveles de nutrientes (Hanek, 1982; Guerra et al.,1990; Bayley et al., 1992; Tello, 1995). Sin embargo, la amplitud, la frecuencia y la duración del flujo de inundación varían entre las cuencas de acuerdo con la gradiente, la topografía, la vegetación y el tipo de suelo. Por ello, los índices de productividad y rendimiento fluctúan considerablemente de un lugar a otro.

\section{Recolección de Datos}

La información recopilada por el Instituto de Investigaciones de la Amazonía Peruana (IIAP), la Dirección Regional de Pesquería de Loreto y la Asociación de Pescadores de Loreto fue utilizada en este estudio. Asimismo, se realizaron trabajos de campo en el puerto y mercado de Iquitos. Esta ciudad de 300000 habitantes, localizada en la región de Loreto, es el principal lugar de comercialización de pescado y el más importante puerto de desembarque de la pesquería comercial de la Amazonía Peruana. Adicionalmente, se hicieron entrevistas a pescadores y a personas vinculadas con la actividad pesquera.

La información se separó en dos grupos para ser analizada: (1) desembarques totales en la región de Loreto y (2) capturas desembarcadas por la flota pesquera comercial en Iquitos. En el primer caso, el pescado salpreso y el seco-salado fueron convertidos a peso fresco multiplicando los valores por 1,8 y 2,5, respectivamente (Hanek, 1982). Los desembarques de ciudades mayores como Nauta, Requena, Contamana y Caballococha fueron incluidos en el primer grupo.

En cuanto a los desembarques del segundo grupo, la información fue recopilada de los registros de viaje de las embarcaciones pesqueras. Desafortunadamente, los datos 
recogidos por los pescadores no mostraron una distinción clara entre las especies del mismo género ni las artes de pesca utilizadas en la captura. Por ello, para analizar los desembarques y los cambios temporales que ocurren en ellos debido a la intensidad de pesca y cambios en el ambiente, las especies fueron agrupadas de acuerdo con su nivel trófico.

Las capturas de la pesca de subsistencia no fueron incluidas en el análisis. Debido a que no fue posible conseguir información sobre descartes, los datos sobre desembarque y captura fueron utilizados indistintamente. Asimismo, cuando el Ucayali y el Amazonas son analizados en conjunto, son referidos como «área de estudio».

\section{Grupos Tróficos}

A pesar de la gran variedad de alimentos consumidos por los peces en la Amazonía, es posible que estos sean clasificados de acuerdo con sus predominantes hábitos alimenticios (Welcomme, 1979; Austen et al., 1994). Tres grupos fueron formados sobre la base de Marlier (1967, 1968), citado por Bayley (1988), y de nuestra observación personal: (1) consumidores primarios o detritívoros, como Prochilodus nigricans, Potamorhina spp. y Curimata spp.; (2) consumidores secundarios u omnívoros, como Colossoma macropomum, Piaractus brachypomus, Brycon spp., Mylossoma spp. y Triportheus spp.; y (3) consumidores terciarios o piscívoros, como Brachyplatystoma flavicans, Brachyplatystoma filamentosum, Pseudoplatystoma fasciatum y Paulicea lutkeni.

Para facilitar el análisis, se utilizaron gráficos en los que se plotearon las capturas por grupos tróficos y por especies, respectivamente, contrastándolas con los años considerados en este estudio (desde 1986 hasta 1996).

\section{RESULTADOS}

\section{Rendimiento Pesquero en la Amazonía Peruana}

El rendimiento pesquero en la Amazonía Peruana fue estimado en $80000 \mathrm{t}$ año ${ }^{1}$ (Bayley et al., 1992; Tello, 1995). La pesquería de subsistencia aportó el 75\% de los desembarques de la región, mientras que el restante $25 \%$ es capturado por la pesquería comercial. Esta última desembarca sus capturas en Loreto (75\%) y Ucayali (25\%), que son las regiones más notables desde el punto de vista pesquero (Figura 1). 
Figura 1. Rendimiento pesquero en la Amazonía Peruana (Bayley et al., 1992).

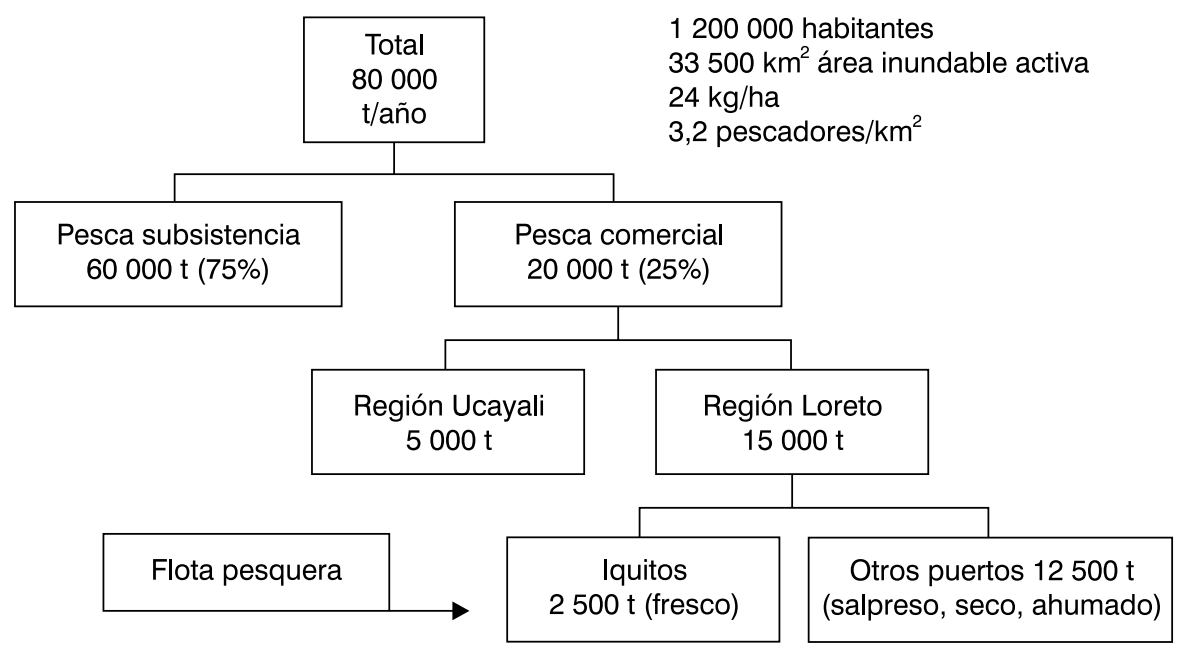

\section{Desembarques en la región Loreto}

La cantidad de pescado desembarcada en la región Loreto durante 1996 fue estimada en 13200 toneladas por la Dirección Regional de Pesquería de Loreto. El 85\% de esta cantidad fue desembarcada en Iquitos, Requena, Nauta, Contamana, Caballococha y Puerto Alegría como fresco, salpreso y seco-salado por embarcaciones que no pertenecían a la flota pesquera de Iquitos. Los botes de la flota comercial con base en Iquitos desembarcaron solo pescado fresco en Puerto Belén, lo que significa el 15\% del total comercializado en la región.

Se estima que son 45 especies las explotadas por la pesquería comercial de Loreto y muchas más por la de subsistencia (Tello, 1995). Las especies del grupo characiformes dominan las capturas con el 83,5\% del total desembarcado en 1996, mientras que los siluriformes (generalmente grandes bagres) constituyen el $7 \%$ de dicho total. La especie más abundante durante 1996 fue Prochilodus nigricans (32\% de los desembarques regionales), seguida de Portamorhina spp. y de Curimata spp. con el 20 y $10 \%$ de los desembarques regionales, respectivamente.

El Ucayali fue la cuenca más productiva, debido a que el 57\% del pescado desembarcado en Loreto en los últimos 15 años fue capturado en este río. De otro lado, el 11\% de dicho total fue capturado en el Amazonas; el 10\%, en el Marañón y el 22\%, en otros ríos (Barthem et al., 1995). 


\section{La Flota Pesquera de Iquitos}

Un promedio de 2200 toneladas de pescado fresco es desembarcado cada año por la flota pesquera de Iquitos. La demanda alimenticia de la población urbana en esta ciudad es cubierta, en gran parte, debido a que los bajos precios del pescado permiten a las personas de reducidos ingresos económicos acceder a una fuente de proteína de excelente calidad. El pescado es más barato que la carne de res, el pollo o el cerdo.

Un número promedio de 75 embarcaciones opera cada año en el área de estudio. Si se considera que cada bote invierte entre seis y siete días haciendo los preparativos para el siguiente viaje, entonces el número promedio de viajes por año es de 12 (Tabla 1).

Tabla 1. Número promedio de días por faena de pesca de acuerdo con el régimen hidrológico estimado para 1994 (Del Águila, 1995).

\begin{tabular}{|c|c|c|c|c|}
\hline Temporada & Prom./días/viaje & Prom./días/pesca & Prom./días/venta & Total \\
\hline Creciente & 14 & 7 & 7 & 28 \\
\hline Vaciante & 8 & 4 & 7 & 19 \\
\hline Promedio & 11 & 6 & 7 & 24 \\
\hline
\end{tabular}

El número de días por viaje depende del tamaño de la embarcación, del ciclo hidrológico y del número de barras de hielo transportado en las cajas isotérmicas, pero usualmente no excede los 30 días. Una unidad de pesca está equipada para recorrer grandes distancias siguiendo a los cardúmenes durante sus movimientos migratorios, los cuales pueden cubrir hasta $600 \mathrm{~km}$. Las embarcaciones no cuentan con equipos sofisticados de detección de peces (eco-sondas).

El arte de pesca más empleado fue la hondera. Esta puede ser de longitud (desde 90 hasta $180 \mathrm{~m}$ ) y altura (desde 25 hasta $35 \mathrm{~m}$ ) variables. El tamaño de malla estirada varía entre 1,5 y 2 pulgadas. Las arrastradoras de playa y las agalleras son utilizadas dependiendo del régimen hidrológico y de la especie seleccionada. Una descripción más detallada de las características de las embarcaciones pesqueras es presentada por Tello (1994).

\section{Desembarques de la Flota Pesquera de Iquitos}

Un total de 40 especies fue explotado por la flota pesquera de Iquitos en los últimos nueve años. Sin embargo, muchas especies más son capturadas, debido a que varias 
del mismo género son registradas solo por un nombre común. De las 2200 toneladas de pescado fresco desembarcado en promedio anualmente en Iquitos, el 58,5\% fue capturado en el Ucayali, el 26\% en el Amazonas, el 6,3\% en el Marañón y el 9,2\% en otros ríos (Tabla 2). Tres especies, Potamorhina spp., Curimata spp. y Prochilodus nigricans, representaron el 62\% del total desembarcado en Iquitos durante 1996.

Tabla 2. Pescado fresco (toneladas) desembarcado anualmente por la flota pesquera de Iquitos.

\begin{tabular}{|c|c|c|c|c|c|}
\hline Año & Ucayali & Amazonas & Marañón & Otros* & Total \\
\hline 86 & 2191 & 264 & 129 & 172 & 2756 \\
87 & 1067 & 1015 & 56 & 144 & 2282 \\
88 & 1172 & 207 & 350 & 330 & 2059 \\
91 & 1165 & 673 & 80 & 262 & 2180 \\
92 & 1429 & 329 & 115 & 325 & 2198 \\
93 & 1210 & 1009 & 92 & 139 & 2450 \\
94 & 634 & 906 & 230 & 137 & 1907 \\
95 & 1216 & 520 & 134 & 102 & 1972 \\
96 & 1508 & 235 & 62 & 170 & 1975 \\
\hline
\end{tabular}

* Putumayo, Tapiche, Tigre, Pastaza, Napo, etc.

\section{Esfuerzo de Pesca}

La más confiable, exacta y sencilla medida del esfuerzo pesquero fue derivada del número de pescadores-viaje por río y por año y del número de viajes por río y por año. El número de días no fue considerado debido a su variabilidad. Si el tiempo de búsqueda de los cardúmenes o el tránsito hacia los lugares de pesca cambia frecuentemente debido a factores tales como la distancia recorrida, el tamaño de la embarcación-caja isotérmica o el ciclo hidrológico, el número de días por viaje será siempre un estimador sesgado del esfuerzo pesquero (Gulland, 1964, citado por Murphy y Willis, 1996). Como no fue posible disponer de información acerca de captura por tipo de red empleado, el esfuerzo de pesca fue utilizado sin tener en cuenta el arte de pesca.

\section{Captura y Esfuerzo de Pesca}

Los datos de esfuerzo fueron usados para estimar los índices de biomasa (CPUE) de la flota pesquera de Iquitos. La captura por unidad de esfuerzo en el área de estudio 
varió de $454 \mathrm{~kg}$ pescador-viaje $\mathrm{e}^{-1}$ en 1986 a $433 \mathrm{~kg}$ pescador-viaje $\mathrm{e}^{-1}$ en 1996 . En cuanto al número de viajes, la CPUE varió de 3,97 t viaje ${ }^{-1}$ en 1986 a 2,94 t viaje en $^{-1} 1996$ (Figura 2).

Durante 1995, ocurrió un fenómeno poco peculiar en el Amazonas. Una migración de $B$. vaillantii acompañada de characiformes apareció en las cercanías de Iquitos. Debido al alto costo por día que representaba mover los botes madrinas para viajes cortos, los pescadores optaron por utilizar los botes auxiliares, de menor capacidad y mayor velocidad. Por ello, el número de pescadores-viaje y el número de viajes se incrementaron significativamente: en $50 \%$ y $110 \%$, respectivamente. El índice de captura expresado como CPUE disminuyó durante ese año.

Figura 2. Índices de biomasa (CPUE) de la flota pesquera de Iquitos.
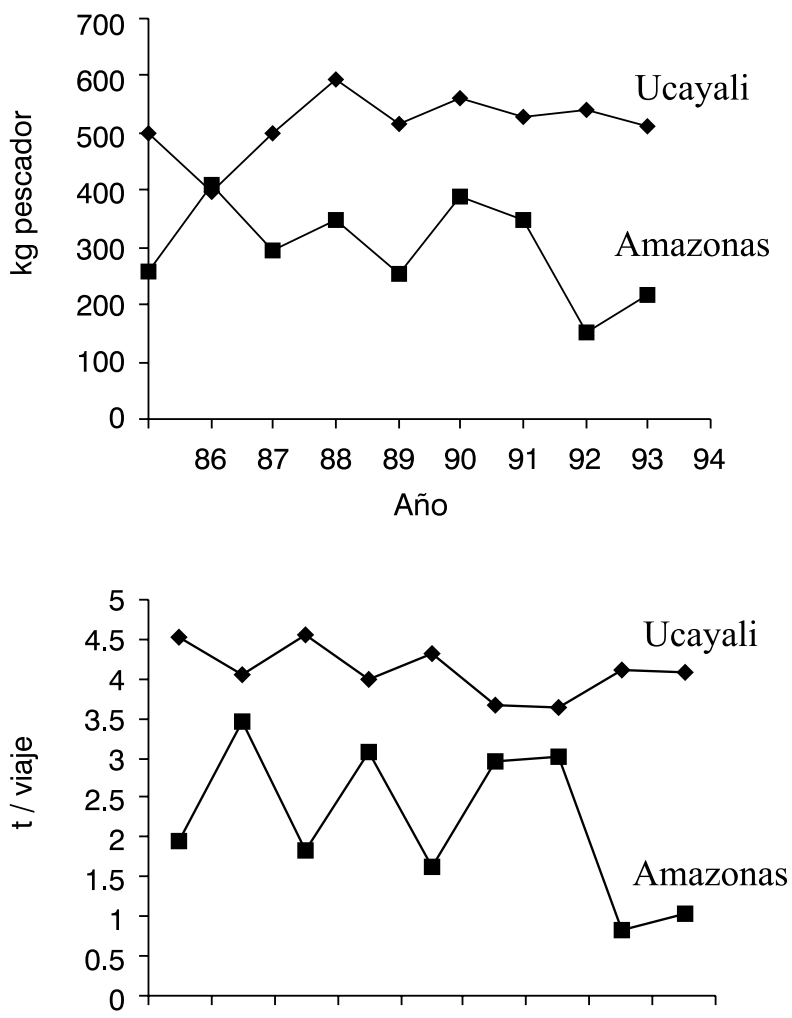

$\begin{array}{lllllllll}86 & 87 & 88 & 89 & 90 & 91 & 92 & 93 & 94\end{array}$

Año 
El índice anual de biomasa, excluyendo el año 1995 en el Amazonas, se incrementó ligeramente en el Ucayali y decreció en el Amazonas durante los años considerados en el análisis. El Ucayali mostró el mayor índice de abundancia (CPUE). Esta cuenca es la más productiva de los sistemas fluviales de planicies inundables en la Amazonía Peruana (Hanek, 1982; Bayley et al., 1992).

Debido a que la aplicación de modelos diseñados para pesquerías monoespecíficas en pesquerías de múltiples especies como la amazónica implica mucho riesgo (Welcomme, 1985), el siguiente análisis debe ser considerado exploratorio. Un análisis de la relación entre rendimiento $\left(\mathrm{kg} \mathrm{ha}^{-1}\right)$ e intensidad de pesca (número de pescadores por kilómetro cuadrado) se llevó a cabo en el área de estudio utilizando el modelo de Graham-Schaefer (Schaefer, 1954; Ricker, 1975). La relación determinada fue:

Yield $\left(\mathrm{kg} \mathrm{ha}^{-1}\right)=-0,4328+7,3185(\mathrm{E})-4,5879(\mathrm{E})^{2}($ estimado de Tabla 3$)$

de la cual:

Nivel óptimo de esfuerzo de pesca (Ricker, 1975) $=\mathrm{a}(2 \mathrm{~b})^{-1}=0,8$ pescadores por $\mathrm{km}^{2}$ $\operatorname{MSY}\left(\right.$ Ricker, 1975) $=\mathrm{a}^{2}(4 \mathrm{~b})^{-1}=2.9 \mathrm{~kg} \mathrm{ha}^{-1}$

donde:

$$
\begin{aligned}
& \mathrm{E}=\text { intensidad de pesca }\left(\text { pescadores } \mathrm{km}^{-2}\right) \\
& \mathrm{a}=\text { coeficiente de } \mathrm{E} \\
& \mathrm{b}=\text { coeficiente de } \mathrm{E}^{2}
\end{aligned}
$$

Tabla 3. Número de pescadores por área de inundación activa y rendimiento pesquero de la flota comercial de Iquitos en el área de estudio.

\begin{tabular}{|c|c|c|}
\hline Año & $\mathrm{N}^{\circ}$ pescadores $\mathrm{km}^{-2}$ & Rendimiento $\left(\mathrm{k} \mathrm{ha}^{-1}\right)$ \\
\hline 1986 & 0,44 & 1,98 \\
1987 & 0,42 & 1,68 \\
1988 & 0,25 & 1,11 \\
1991 & 0,31 & 1,48 \\
1992 & 0,33 & 1,42 \\
1993 & 0,38 & 1,79 \\
1994 & 0,28 & 1,24 \\
1995 & 0,46 & 1,40 \\
1996 & 0,32 & 1,40 \\
\hline
\end{tabular}


De acuerdo con esta relación, el rendimiento total de la flota pesquera de Iquitos en el área de estudio parece incrementarse cuando aumenta el número de pescadores hasta una densidad máxima de cerca de 0,8 pescadores por kilómetro cuadrado. Después de ello, el rendimiento tenderá a declinar conforme se intensifique la explotación.

\section{Cambios en la composición de especies en los desembarques}

En los últimos años, hubo un reemplazo progresivo de especies grandes por especies más productivas y pequeñas en los desembarques regionales. La pesquería en Loreto es sostenida en su mayoría por detritívoros, que representaron el $65 \%$ de los desembarques totales durante 1996. El mayor desembarque de detritívoros ocurrió en 1987 (8 855 t). Esta cifra decreció en $20 \%$ y $50 \%$ durante 1988 y 1989, respectivamente (Figura 3a). A pesar de las variaciones observadas, hay una tendencia positiva de los desembarques de detritívoros en Loreto. P. nigricans fue la especie más abundante en las capturas regionales.

De la misma manera, la captura de omnívoros alcanzó su máximo valor en 1991 (2 148 t) para luego disminuir en 50\%. Probablemente, esto se haya debido a la intensidad de la pesca. Los desembarques de este grupo han disminuido en los últimos cinco años (Figura 3b).

El máximo desembarque de piscívoros ocurrió en 1988 y disminuyó en los años posteriores (Figura 3b). Las especies de este grupo han sido intensamente explotadas en la Amazonía Peruana desde 1980 como consecuencia de dos factores: (1) la introducción de redes agalleras de deriva de grandes dimensiones y (2) la apertura del mercado internacional. Los rendimientos de $B$. flavicans y $P$. fasciatum disminuyeron en el área de estudio en los últimos años (Figura 3c). Las tallas promedio de captura de dorado se han reducido hasta un punto tal que los pescadores están capturando, por lo general, individuos inmaduros. Esta situación podría afectar seriamente al reclutamiento y regeneración de esta valiosa especie (Tello et al., 1995; García et al., 1996). En el caso de los piscívoros, fue imposible conseguir información confiable acerca de la captura en los ríos y/o por artes de pesca, debido a que la pesca de los grandes bagres es realizada por un grupo particular de pescadores sin ninguna relación con la flota pesquera descrita en el presente reporte.

Cambios en la composición de los desembarques de la flota pesquera con base en Iquitos han ocurrido en el grupo de los omnívoros. Una reducción progresiva de especies grandes (Colossoma macropomum y Brycon spp.) y el correspondiente incremento de especies pequeñas, de alto rendimiento, rápido crecimiento y menor precio, como Mylossoma spp. y Triportheus spp. han ocurrido en los desembarques 
regionales, debido probablemente a la intensidad de la pesca. Ello es consecuencia de una mayor demanda en el mercado por la calidad y rendimiento de su carne (Figuras $4 a \mathrm{y} b)$.

Mientras que, en nivel regional, hay una tendencia positiva en los desembarques de detritívoros, controlada generalmente por Prochilodus nigricans, en los desembarques de la flota pesquera de Iquitos, la cantidad de esta especie se ha reducido en los últimos cuatro años. Esto se debe probablemente a una mayor intensidad de pesca en lugares cercanos a las ciudades grandes como Iquitos, Requena, Nauta y Contamana. En los desembarques regionales, no se observa esta tendencia porque el pescado procede no solo del Ucayali y del Amazonas, sino también de otros ríos distantes de las ciudades mencionadas. Asimismo, una reducción progresiva de especies como P. nigricans y el incremento de especies más pequeñas y baratas como Potamorhina spp. se observó en los desembarques de la flota pesquera de Iquitos (Figura 5).

Figura 3.
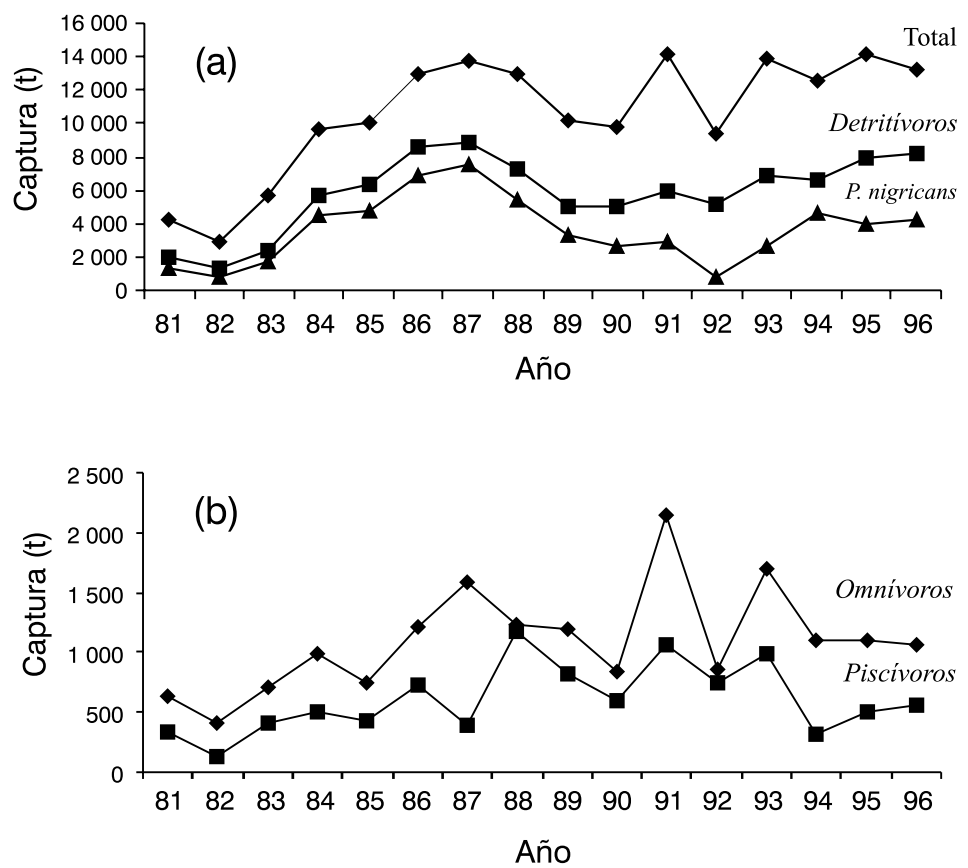


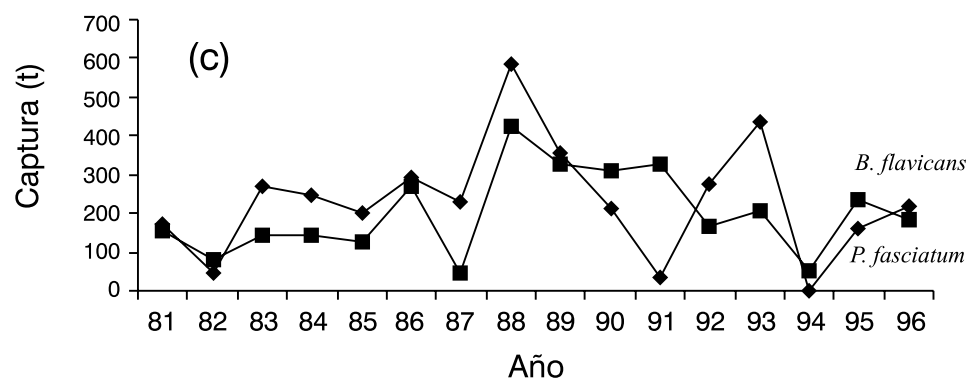

Figura 4. Tendencia histórica de los desembarques en la región Loreto.
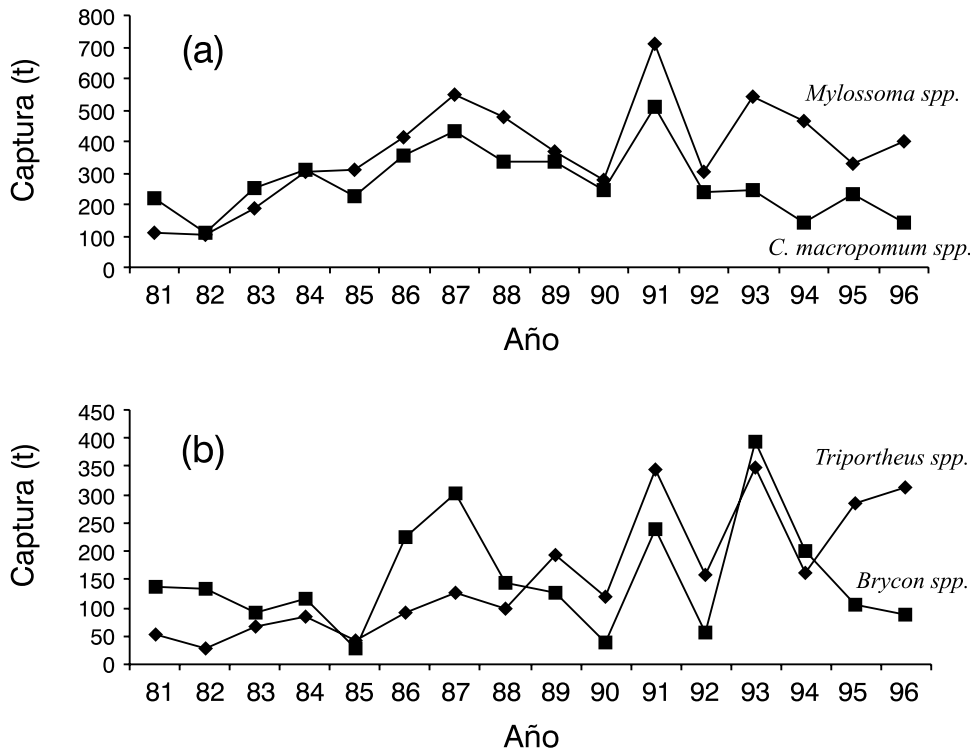
Figura 5. Tendencia histórica de los desembarques de la flota pesquera de Iquitos.

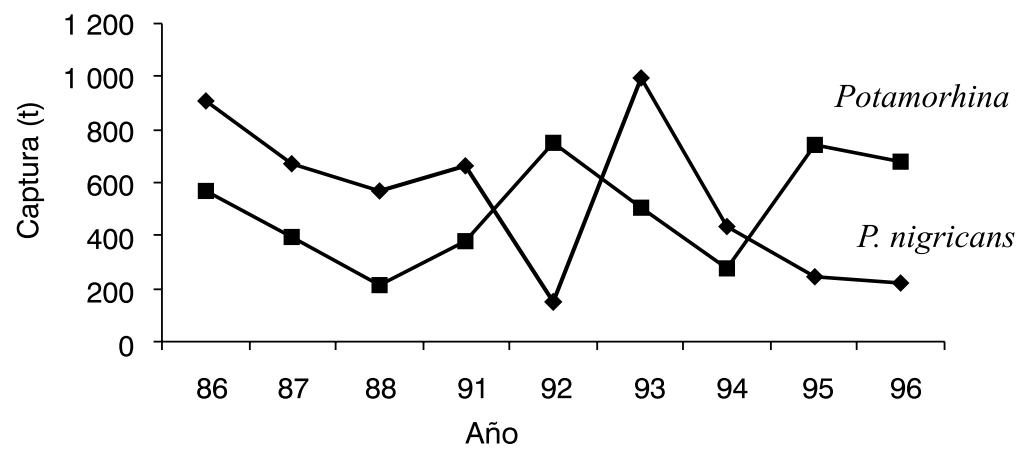

\section{DISCUSIÓN Y CONCLUSIONES}

En la región Loreto, como consecuencia de una mayor intensidad de pesca y de cambios en los tipos de artes de pesca, las especies grandes están siendo reemplazadas en los desembarques por especies más pequeñas, de alto rendimiento y de menor precio. Este proceso selectivo es debido a la preferencia tanto de los pescadores como de los consumidores por las especies grandes, así como también a la baja capacidad de estas especies para soportar altos niveles de mortalidad por pesca (Welcomme, 1985). Cuando la pesca se intensifica, las especies de mayor tamaño son sobre-explotadas y desplazadas progresivamente, en términos de biomasa, por las especies más pequeñas y de vida corta. Esto ocurre, probablemente, porque las especies con ciclo de vida corto se adaptan mejor a la pesca intensiva como consecuencia de altos índices de producción/biomasa. Las especies grandes son menos productivas por unidad de biomasa que las especies más pequeñas (Regier y Henderson, 1973; Turner, 1985; Lowe-McConnell, 1987; Lae, 1995).

En muchos sistemas fluviales de planicies inundables, han ocurrido cambios en la composición de las especies. Novoa (1989) observó la reducción de la talla media y de la proporción de capturas de grandes bagres en el río Orinoco. Asimismo, Bayley y Petrere (1989) encontraron evidencia de la desaparición de especies grandes como A. gigas y C. macropomum en las capturas de la flota pesquera de Manaos, Brasil.

La explotación de piscívoros puede ocasionar el incremento de la producción de especies presas en algunos sistemas fluviales de planicies inundables. Esta tendencia 
fue observada en el Lago Victoria y en el río Nilo, lugares en donde las especies de mayor tamaño fueron reemplazadas por una secuencia de especies de menor tamaño y más productivas (Marten, 1979; Lae, 1995). Comportamientos similares fueron observados en los lagos Malawi y Tanganyika (Turner, 1985).

A pesar de la evidencia de sobre-pesca de crecimiento en algunas especies de grandes bagres, solo futuras observaciones determinarán si esas tendencias son simplemente fluctuaciones o problemas de extinción comercial de algunas poblaciones de peces en la Amazonía Peruana.

En contraste con los resultados encontrados en este estudio para 1996 de un esfuerzo de 0,3 pescadores por $\mathrm{km}^{2}$, con un rendimiento de $1,4 \mathrm{~kg} \mathrm{ha}^{-1}$ (Tabla 3), Petrere (1983) encontró, para la flota pesquera de Manaos, Brasil, un esfuerzo de 0,72 pescadores por $\mathrm{km}^{2} \mathrm{y}$ un rendimiento de $1,74 \mathrm{~kg} \mathrm{ha}^{-1}$, más del doble del número de pescadores para un rendimiento similar estimado hace 18 años. Esta diferencia puede deberse a que, en el Amazonas brasileño, las poblaciones de peces fueron y son sometidas a una mayor intensidad de pesca como consecuencia de una mayor presión del mercado. Por otro lado, el rendimiento pesquero estimado en este estudio es bajo si lo comparamos con los valores estimados por Welcomme (1985) y Bayley y Petrere (1989). Sin embargo, los resultados encontrados por estos autores se basaron en datos que incluían no solo la pesca comercial, como es el caso nuestro, sino también la pesca de subsistencia.

La idea de que las pesquerías de alto rendimiento pueden, probablemente, sostener estos niveles de producción bajo las condiciones actuales de explotación es, en parte, planteada por este estudio. Los rendimientos comerciales a similares niveles de esfuerzo encontrados en el área de estudio han permanecido casi constantes a pesar de los cambios en la composición de las capturas detectados en los últimos años.

\section{BIBLIOGRAFÍA}

AUSTEN, D. J.; BAYLEY, P.B.; MENZEL, B.W. 1994. Importance of the guild concept to fisheries research and management. En: Fisheries 6:12-20.

BARTHEM, R. H.; GUERRA, M.; VALDERRAMA. 1995. Diagnóstico de los recursos hidrobiológicos de la Amazonía. En: Tratado de Cooperación Amazónica (TCA). Secretaría Pro Tempore. 142 pp. 
BAYLEY, P.B. 1988. Factors affecting growth rates of young tropical floodplain fishes: seasonality and density-dependence. En: Environmental Biology of Fishes 21(2): 127-142.

BAYLEY, P.B.; PETRERE, M. 1989. Amazon Fisheries: Assessment methods, current status and management options. En: Dodge, P. (ed.). Proceeding of the International Large River Symposium. Canadian Special Publication of Fisheries and Aquatic Sciences 106: 385-398.

BAYLEY, P.B.; VÁSQUEZ, P.; GHERSI, F.; SOINI, P.; PINEDO, M. 1992. Environmental review of the Pacaya-Samiria National Reserve in Peru and assessment of project. An environmental assessment contract completed for the Nature Conservancy. $78 \mathrm{pp}$.

DEL ÁGUILA, R. 1995. Descripción de la pesquería comercial con base en Iquitos. Iquitos (Perú): Universidad Nacional de la Amazonía Peruana. Tesis de Biólogo.

GARCÍA, A.; RODRÍGUEZ, R.; MONTREUIL, V. 1996. Longitud de primera maduración y época de desove de dorado, B flavicans, en la Amazonía Peruana. En: Boletín Científico No4 /15-17/. Santa Fe de Bogotá (Colombia).

GUERRA, H.; ALCÁNTARA, F.; MACO, J.; SÁNCHEZ, H. 1990. La pesquería en el Amazonas peruano. En: Interciencia 6: 469-475.

GULLAND, J.A. 1982. The management of tropical multispecies fisheries. En: Pauly, D. y Murphy, G.Y. (ed.). Theory and Management of Tropical Fisheries. Conference proceeding 9: 287-298.

HANEK, G. 1982. La pesquería en la Amazonía Peruana: presente y futuro. FAO. En: Documentos Técnicos de Pesca 81: 350 pp.

LAE, R. 1995. Climatic and anthropogenetic effects on fish diversity and fish yields in the Central Delta of the Niger River. En: Aquatic Living Resource 8: $43-58$.

LOWE McCONNELL, R.H. 1987. Ecological studies in tropical fish communities. En: Cambridge Tropical Biology Series: Cambridge University Press, $382 \mathrm{pp}$. 
MARTEN, G. 1979. Impact of fishing on the inshore fishery of Lake Victoria (East of Africa). En: Journal Fishery Research Board Canadian 34: 891-900.

MONTREUIL, V.; TELLO, S. 1990. Rendimiento máximo sostenible de la pesquería comercial de Loreto. En: Fishbyte 8, ICLARM, Philippines.

1997. Rendimiento máximo sostenible de la pesquería comercial de boquichico, P. nigricans. Congreso sobre manejo de fauna silvestre en la Amazonía Peruana, Iquitos (Perú).

MURPHY, B.; WILLIS, D. 1996. Fisheries techniques. Maryland (USA): American Fisheries Society. $732 \mathrm{pp}$.

NOVOA. D. 1989. The multispecies fisheries of the Orinoco River: Development, present status, and management strategies. En: Canadian Special Publication of Fisheries and Aquatic Sciences 106:422-428.

PETRERE, M. 1983. Relationships among catches, fishing effort and river morphology for eight rivers in Amazonas State (Brazil), during 1976-1978. En: Amazoniana 8: 281 - 296.

REGIER, H.; HENDERSON, H. 1973. Towards a broad ecological model of fish communities and fisheries. En: Transactions of the American Fisheries Society 12(1): 56-72.

RICKER, W.E. 1975. Computation and interpretation of biological statistics of fish populations. En: Bulletin of Fishery Researches Board Canada 191. $382 \mathrm{pp}$.

SCHAEFER, M.B. 1954. Some aspects of population dynamics for the management of commercial marine fishery. En: Bulletin of the Inter American Tropical Tuna Commission: 25-56.

TELLO, S. 1994. Características de la flota pesquera comercial de Iquitos, Perú. En: Folia Amazónica 6 (1-2), IIAP. Iquitos (Perú).

TELLO, S.; GUERRA, H.; GARCÍA, A.; RODRÍGUEZ, R. 1995. La pesquería de grandes bagres en la región Loreto con énfasis en dorado, B. flavicans. En: Informe Técnico IIAP. Iquitos (Perú). 
TELLO, S. 1995. Relevamiento de información sobre captura y esfuerzo pesquero con destino a ciudades. En: Documento Técnico 12 IIAP. Iquitos (Perú).

TURNER, J.L. 1985. Changes in multispecies fisheries when many species are caught at the same time. En: FAO Fisheries Report. 338: 201-211.

WELCOMME, R.L. 1975. The fisheries ecology of African floodplains. Comitee for Inland Fisheries of Africa (FAO). En: CIFA Technical Paper 3. 51 pp. 1979. Fisheries ecology of floodplain rivers. Welcomme (ed.). Longman, London and N.Y.

1985. River fisheries. En: FAO Fisheries Technical Paper 262. 327 pp. 